\title{
Reduced epithelial suppressor of cytokine signalling 1 in severe eosinophilic asthma
}

\author{
Emma Doran ${ }^{1}$, David F. Choy ${ }^{2}$, Aarti Shikotra ${ }^{3}$, Claire A. Butler ${ }^{1}$, \\ Declan M. O'Rourke ${ }^{4}$, James A. Johnston ${ }^{5}$, Adrien Kissenpfennig ${ }^{1}$, \\ Peter Bradding ${ }^{3}$, Joseph R. Arron ${ }^{2}$ and Liam G. Heaney ${ }^{1}$
}

Affiliations: ${ }^{1}$ Centre for Infection and Immunity, Health Sciences Building, Queens University Belfast, Belfast, UK ${ }^{2}$ ITGR Diagnostic Discovery, Genentech, San Francisco, CA, USA. ${ }^{3}$ Department of Infection, Immunity and Inflammation, Maurice Shock Medical Sciences Building, Leicester, UK. ${ }^{4 H i s t o p a t h o l o g y}$ and Cytopathology Laboratory, Belfast Health and Social Care Trust, Belfast, UK. ${ }^{5}$ Inflammation, Amgen Inc, Thousand Oaks, CA, USA.

Correspondence: Liam Heaney, Centre for Infection and Immunity, Health Sciences Building, Queens University Belfast, Lisburn Road, BT9 7AE, Northern Ireland, UK. E-mail: I.heaneyqqub.ac.uk

ABSTRACT Severe asthma represents a major unmet clinical need. Eosinophilic inflammation persists in the airways of many patients with uncontrolled asthma, despite high-dose inhaled corticosteroid therapy. Suppressors of cytokine signalling (SOCS) are a family of molecules involved in the regulation of cytokine signalling via inhibition of the Janus kinase-signal transducers and activators of transcription pathway. We examined SOCS expression in the airways of asthma patients and investigated whether this is associated with persistent eosinophilia.

Healthy controls, mild/moderate asthmatics and severe asthmatics were studied. Whole genome expression profiling, quantitative PCR and immunohistochemical analysis were used to examine expression of SOCS1, SOCS2 and SOCS3 in bronchial biopsies. Bronchial epithelial cells were utilised to examine the role of SOCS1 in regulating interleukin (IL)-13 signalling in vitro.

SOCS1 gene expression was significantly lower in the airways of severe asthmatics compared with mild/ moderate asthmatics, and was inversely associated with airway eosinophilia and other measures of T-helper type 2 (Th2) inflammation. Immunohistochemistry demonstrated SOCS1 was predominantly localised to the bronchial epithelium. SOCS1 overexpression inhibited IL-13-mediated chemokine ligand (CCL) 26 (eotaxin-3) mRNA expression in bronchial epithelial cells.

Severe asthma patients with persistent airway eosinophilia and Th2 inflammation have reduced airway epithelial SOCS1 expression. SOCS1 inhibits epithelial IL-13 signalling, supporting its key role in regulating Th2-driven eosinophilia in severe asthma.

@ERSpublications

Persistent airway eosinophilia/Th2 inflammation in severe asthma is linked to reduced epithelial SOCS1 expression http://ow.ly/Mlgl3001UMU

Editorial comment in Eur Respir J 2016; 48: 608-610.

This article has supplementary material available from erj.ersjournals.com

Received: March 112015 | Accepted after revision: April 242016 | First published online: June 232016

Support statement: This research was supported by a $\mathrm{PhD}$ studentship from the Department of Employment and Learning, Northern Ireland; grant support from Northern Ireland Chest Heart and Stroke; and grant support from Genentech Inc and the National Institute for Health Research Leicester Respiratory Biomedical Research Unit. The views expressed are those of the authors and not necessarily those of the NHS, the NIHR, or the Department of Health. Funding information for this article has been deposited with the Open Funder Registry.

Conflict of interest: Disclosures can be found alongside this article at erj.ersjournals.com

Copyright @ERS 2016 


\section{Introduction}

Asthma is one of the most common chronic conditions affecting approximately 300 million people worldwide [1]. Most patients with asthma achieve adequate disease control with bronchodilator and inhaled corticosteroid therapy [2]. However, approximately $10 \%$ of patients suffer from severe uncontrolled asthma despite the use of high-dose inhaled corticosteroids and, in many cases, systemic corticosteroids. This cohort of asthma patients represents a significant unmet clinical need [3].

Asthma is a heterogeneous disorder thus current research is endeavouring to identify and characterise distinct phenotypes, particularly in severe disease $[4,5]$. Both clinical and molecular phenotyping have been utilised to describe populations of asthma patients in terms of inflammatory biology [6-8].

A key feature in many patients with severe asthma is persistent eosinophilic airway inflammation despite high-dose corticosteroid treatment $[9,10]$. The major group of cytokines implicated in driving this inflammation are the T-helper type 2-associated cytokines, namely interleukin (IL)-4, IL-5 and IL-13. IL-13 is a key cytokine contributing to the pathophysiological features of asthma including airway hyper-responsiveness, mucus hypersecretion and airway remodeling, and IL-13 overexpression persists in a subgroup of patients with severe asthma despite high-dose steroid treatment $[10,11]$.

Th2 cytokines mediate their actions via the Janus kinase-signal transducers and activators of transcription (JAK-STAT) pathway and cytokine signalling is tightly regulated by several molecules including the suppressors of cytokine signalling (SOCS). SOCS are a family of eight proteins, comprising SOCS1-7 and cytokine-inducible SH2-containing protein (CIS), which inhibit cytokine signalling through inhibition of the JAK-STAT pathway in a negative feedback manner [12]. SOCS1 was the first of the SOCS family to be discovered [13]. In vitro cell cultures and in vivo animal models have demonstrated that SOCS1 is a negative regulator of Th2-dependent pathways, achieved by inhibition of the phosphorylation of signal transducer and activator of transcription (STAT) 6 [14, 15]. SOCS2 inhibits growth hormone and insulin-like growth factor [16] and has been shown to regulate the expression of other SOCS molecules [17]. Recently, we have shown that SOCS2 knockout mice have an increased susceptibility to Th2-mediated airway inflammation compared to wild-type mice, due to elevated SOCS3 levels [18]. SOCS3 mRNA expression is reported to be increased in the peripheral T cells of asthma patients $[19,20]$ and constitutive SOCS3 expression in the T cells of mice resulted in exaggerated lung inflammation in a mouse model of asthma [20].

The principal aim of this study was to examine SOCS1, SOCS2 and SOCS3 expression in the airways of healthy controls, mild/moderate asthmatics and severe asthmatics and to explore the relationship between SOCS expression and persistent eosinophilic inflammation within the airway.

\section{Methods}

\section{Patient cohort}

Data from this well-characterised cohort of subjects has been published previously [10, 21, 22]. Healthy controls $(n=17)$, mild/moderate asthmatics $(n=29)$ and severe asthmatics $(n=18)$ were recruited from two specialist asthma centres, Belfast City Hospital, Belfast, UK, and Glenfield Hospital, Leicester, UK (demographic characteristics shown in table 1). Clinical assessment protocols ensured patients had severe asthma as defined by current European Respiratory Society/American Thoracic Society guidelines [23]. Further details of asthma patients in the study, including asthma treatment, can be found in the online supplementary file (table E1). This study was approved by Research Ethics Committees of both institutions and written informed consent obtained from all subjects (see online supplementary file).

\section{Bronchoscopy}

Each participant underwent bronchoscopy and biopsy specimens were taken from the right middle lobe and lower lobe carinae. Biopsies were immediately placed in RNA preservative (Ambion; ThermoFisher Scientific, Waltham, MA, USA) or fixed overnight before embedding in glycol methacrylate as described previously [24].

\section{Gene expression analyses}

Bronchial biopsies were homogenised, RNA extracted and amplified for two-colour Whole Human Genome $4 \times 44 \mathrm{k}$ gene expression microarray analysis (Agilent, Santa Clara, CA, USA) as previously described [25]. Th2 score was calculated as described previously and details can be found in the online supplementary file [8, 10, 25]. TaqMan Gene Expression Assays (Applied Biosystems, Waltham, MA, USA) were used as per manufacturer's instructions. GAPDH was utilised as the house keeping gene. Quantitative PCR values were calculated using the $\Delta \Delta \mathrm{Ct}$ method with relative gene expression calculated as $-\Delta \Delta \mathrm{Ct}$ [26]. Further details are available in the online supplementary file. 
TABLE 1 Demographic characteristics of study participants

\begin{tabular}{|c|c|c|c|c|}
\hline & Healthy controls & Mild-moderate asthmatics & Severe asthmatics & $\mathrm{p}$-value \\
\hline Participants & 17 & 29 & 18 & \\
\hline Age years & $31.82 \pm 11.74$ & $31.86 \pm 9.93$ & $46.94 \pm 10.70$ & $<0.0001$ \\
\hline Duration of asthma years & NA & $14.59 \pm 13.76$ & $19.39 \pm 15.01$ & NS \\
\hline Asthma onset age & NA & $17.28 \pm 13.29$ & $27.56 \pm 18.99$ & 0.03 \\
\hline FEV 1 L & $3.69 \pm 0.62$ & $3.48 \pm 0.73$ & $2.45 \pm 0.60$ & $<0.0001$ \\
\hline Mean asthma exacerbations per year & NA & $2.2 \pm 0.83$ & $5.26 \pm 4.14$ & $<0.0001$ \\
\hline Regular inhaled steroid & NA & $20(69 \%)$ & $18(100 \%)$ & NS \\
\hline BDP equivalent $\mu \mathrm{g}$ & NA & $400(0-800)$ & $1600(800-1700)$ & $<0.0001$ \\
\hline Systemic steroids & NA & $0(0 \%)$ & $12(67 \%)$ & $<0.0001$ \\
\hline Prior smoking & $1(6)$ & $6(21 \%)$ & $7(39 \%)$ & NS \\
\hline
\end{tabular}

Data presented as mean \pm SD or median (interquartile range), unless otherwise stated. Between group comparisons were calculated using ANOVA with Bonferroni's multiple comparisons test or Kruskal Wallis with Dunn's multiple comparison test where appropriate; $t$-test when only asthma groups compared or Chi-squared for categorical variables. FEV1: forced expiratory volume in 1 s; FVC: forced vital capacity; BDP: beclomethasone dipropionate; NA: not applicable; Ns: not significant.

\begin{abstract}
Immunohistochemistry
Sections of bronchial biopsies were cut at $2 \mu \mathrm{m}$, immunostained as described previously [24] and counterstained with Mayer's haematoxylin. Negative controls, using an isotype and/or omitting primary antibody, were employed. The lamina propria was measured in biopsy sections using a computer analysis system (LAS V3.7 software, Leica Microsystems, Wetzlar, Germany). For enumerating cellular inflammatory infiltrate, individual positively stained nucleated cells were counted and expressed per $\mathrm{mm}^{2}$ of tissue. Figure E1 in the online supplementary file shows exemplar micrographs of inflammatory cell staining. Immunohistochemistry (IHC) was additionally employed to determine localisation of SOCS proteins in patient biopsies. Full details of antibodies are available in the online supplementary file.
\end{abstract}

\section{Bronchial epithelial cell cultures}

The BEAS-2B cell line (ATCC, Manassas, VA, USA) was cultured in Dulbecco's Modified Eagle Medium supplemented with $5 \%$ fetal calf serum and $1 \%$ penicillin/streptomycin. Primary bronchial epithelial cells (PBECs) were obtained from healthy volunteers. This work was approved by Research Ethics Committee as detailed in the online supplementary file and written informed consent was gained from all subjects [22]. PBECs were cultured in airway epithelial cell basal medium containing a supplement pack (Promocell, Heidelberg, Germany). Human recombinant IL-13 (R\&D Systems, Minneapolis, MN, USA) was used at $50 \mathrm{ng} \cdot \mathrm{mL}^{-1}$. In vitro experiments were carried out a minimum of three times.

\section{Transfections}

Myc-tagged full-length SOCS1 in a pcDNA3 plasmid, along with the empty vector, were a generous gift from A. Yoshimura (Keio University School of Medicine, Tokyo, Japan). BEAS-2B cells were plated in antibiotic-free media and transfected using Lipofectamine 2000 (ThermoFisher). PBECs were transfected by electroporation using the NEON transfection system (ThermoFisher). Media was changed $6 \mathrm{~h}$ post-transfection and cell stimulations were carried out $24 \mathrm{~h}$ post-transfection.

\section{Western blotting}

Cells were lysed and $30 \mu \mathrm{g}$ of protein was immunoblotted with antibodies against SOCS1, SOCS2, SOCS3, c-Myc and $\gamma$-tubulin as described in the online supplementary file.

\section{Statistical analyses}

Prism 5 software (GraphPad, San Diego, CA, USA) was used to analyse data. Data are presented as median (interquartile range) where appropriate. Kruskal-Wallis test, Mann-Whitney U-test, T-tests and Spearman's Rank correlations were used for statistical tests unless otherwise stated. PBEC data was transformed $(\log 10)$ prior to statistical analysis. 


\section{Results}

Increased Th2 inflammatory measures in study participants with asthma

Peripheral blood eosinophil numbers and blood IgE levels were significantly elevated in mild/moderate asthmatics and severe asthmatics compared with healthy controls (figure $1 \mathrm{a}$ and $\mathrm{b}$, respectively). Quantification of eosinophils using IHC showed a trend of increasing numbers of eosinophils in the lamina propria of bronchial biopsies with increasing asthma severity (healthy control, mild/moderate asthma and severe asthma group median values were 4.59, 9.70 and 12.93 respectively; $\mathrm{p}=0.07$ ) (figure $1 \mathrm{c}$ ). There were no significant differences noted in the number of neutrophils or mast cells between the study groups (table E2 in the online supplementary file). The previously generated gene expression microarray Th2 signature from asthma patients is a composite measure of IL-13-induced epithelial genes [8, 10, 25]. There was also a trend for increasing Th2 biopsy signature values with increasing asthma severity $(\mathrm{p}=0.057)$. There was a significant positive correlation between the intensity of the Th2 biopsy signature and the number of eosinophils $/ \mathrm{mm}^{2}$ of lamina propria of the asthma patients (figure 1e). No correlation was observed between neutrophils or mast cells and the Th2 signature (figure E2 in the online supplementary file).

\section{Decreased SOCS1 mRNA expression in bronchial biopsies from severe asthmatics compared with mild/moderate asthmatics}

Gene expression of SOCS1, SOCS2 and SOCS3 mRNA in bronchial biopsies of healthy controls, mild/moderate asthmatics and severe asthmatics was assessed using quantitative PCR. SOCS1 expression was significantly decreased in the bronchial biopsies of severe asthmatics compared to mild/moderate asthmatics (figure 2a). However, there were no differences observed in SOCS2 and SOCS3 gene expression levels between groups (figure $2 \mathrm{~b}$ and $\mathrm{c}$, respectively). Of note, SOCS1 mRNA was decreased in the airways of asthma patients receiving high-dose inhaled corticosteroids compared those receiving low-dose inhaled corticosteroids but there was no difference in SOCS2 or SOCS3 expression (figure E3).
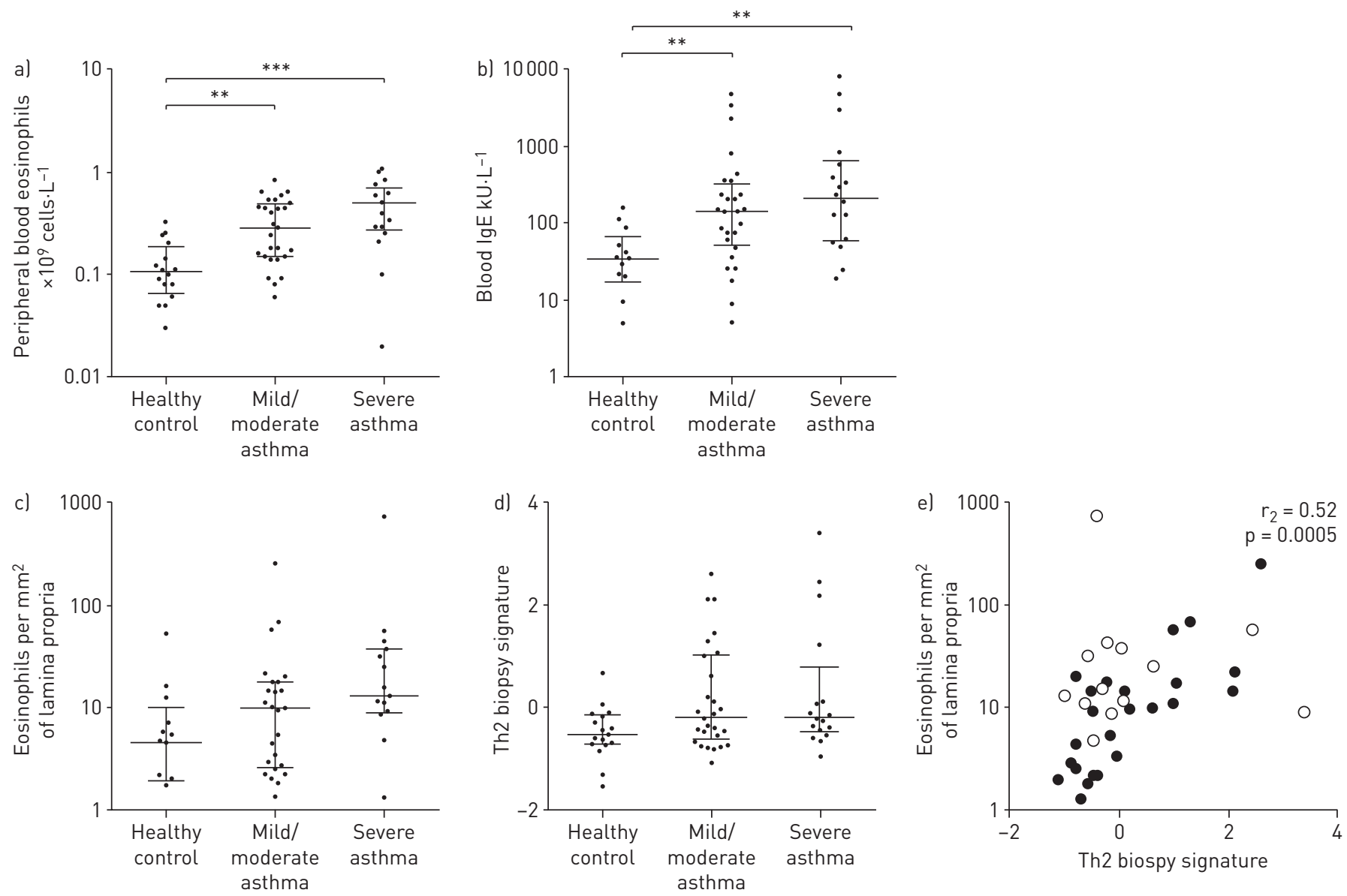

FIGURE 1 Increased T-helper type 2 (Th2) inflammatory measures in study participants with asthma. a) Peripheral blood eosinophils and b) blood IgE levels increased with increasing asthma severity. $c$ and d) A trend of increasing eosinophil numbers in the lamina propria and increasing Th2 signature values with increasing asthma severity was observed. e) Eosinophil numbers in the lamina propria positively correlated with Th2 biopsy signature $(n=40)$. Black circles represent mild/moderate asthma patients and white circles represent severe asthma patients. Kruskal-Wallis and Spearman's rank tests were employed for statistical analysis. Data are presented as median (interquartile range). ${ }^{* *}: p<0.01 ;{ }^{* * *}: p<0.001$. 

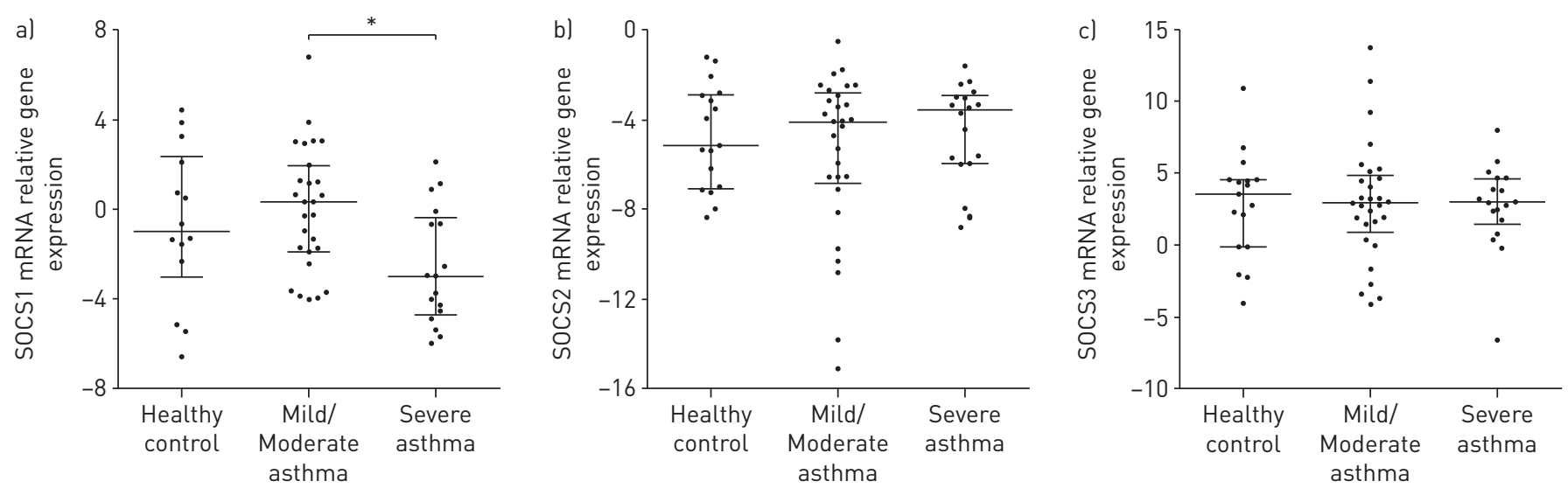

FIGURE 2 Decreased suppressor of cytokine signalling (SOCS)1 mRNA expression in bronchial biopsies from severe asthmatics compared to mild/moderate asthmatics. a) SOCS1, b) SOCS2 and c) SOCS3 mRNA expression was analysed in biopsy homogenates from healthy controls $(n=17)$, mild/moderate asthmatics $(n=29)$ and severe asthmatics $(n=18)$ using quantitative PCR. Values represent $-\Delta \Delta C t$. The Kruskal-Wallis test was employed for statistical analysis. Data are presented as median (interquartile range). *: $p<0.05$.

\section{SOCS1 gene expression is decreased in patients with persistent Th2 inflammation and} eosinophilia in the airways

Negative correlations were observed between SOCS1 gene expression and both the Th2 biopsy signature and eosinophil numbers in the lamina propria of bronchial tissue in asthma patients (figure $3 a$ and $b$, respectively). There were no significant correlations identified between SOCS2 or SOCS3 and Th2 biopsy signature or eosinophil numbers in bronchial biopsies (figure E4 in the online supplementary file). To further examine the relationship between SOCS1 expression and tissue eosinophilia, subjects were dichotomised by the number of eosinophils in the lamina propria of their bronchial biopsies (eosinophil counts were dichotomised around the median value for eosinophils per $\mathrm{mm}^{2}$ within the lamina propria). Participants with high eosinophils $\left(\geqslant 9.2\right.$ eosinophils $\cdot \mathrm{mm}^{-2}$ within the lamina propria) were found to have significantly decreased levels of SOCS1 when compared to those presenting with low eosinophils $\left(<9.2\right.$ eosinophils $\cdot \mathrm{mm}^{-2}$ within the lamina propria) (figure 3c). Subjects dichotomised into eosinophil high and eosinophil low subsets again showed no difference in SOCS2 or SOCS3 expression between the two groups (figure E5 in the online supplementary file). As chemokine ligand (CCL) 26 is highly induced in response to type 2 inflammation, the relationship between this chemokine and SOCS1 was explored. As anticipated, a negative correlation was observed indicating patients with low SOCS1 expression fail to inhibit the Th2 induced CCL26 (figure 3d).

\section{Localisation of SOCS proteins in bronchial biopsies}

To examine the cellular localisation of SOCS protein expression, IHC was employed to stain for SOCS1, SOCS2 and SOCS3 in the bronchial biopsies. SOCS1 was localised predominantly to the bronchial epithelium and observed in the differentiated pseudo-columnar cells rather than the basal cells (figure 4 shows representative images of: a) negative control staining; and SOCS1 staining of b) healthy controls, c) mild/moderate asthmatics and d) severe asthmatics). There was minimal SOCS2 staining evident throughout the bronchial biopsies with only a small number of infiltrating inflammatory cells in the lamina propria staining positive for SOCS2 (figure E6 in the online supplementary file). SOCS3 was found to be present both in the epithelium and in large, mononuclear/macrophage-like cells in the lamina propria (figure E6 in the online supplementary file).

\section{Inhibition of IL-13 induced CCL26 mRNA in bronchial epithelial cell SOCS1 overexpression models} Due to the localisation of SOCS1 to the bronchial epithelium and its potential role in inhibiting Th2 inflammation within the severe asthmatic airway, the BEAS-2B cell line was initially used to further characterise the role of SOCS1 in IL-13 signalling in the bronchial epithelium. We examined induction of SOCS1 mRNA in BEAS-2B cells after IL-13 (50 $\left.\mathrm{ng} \cdot \mathrm{mL}^{-1}\right)$ exposure and found it was rapidly up-regulated within $2 \mathrm{~h}$ of stimulation and remained elevated over the $24 \mathrm{~h}$ time course. SOCS2 and SOCS3 mRNA were not induced in response to IL-13 treatment (figure 5a). We then investigated the expression of SOCS1, SOCS2 and SOCS3 protein in response to IL-13 stimulation observing SOCS1 induction (increase in SOCS1 $/ \gamma$-tubulin ratio from 1 at $0 \mathrm{~h}$ to 1.3 at $24 \mathrm{~h}$ ) but not SOCS2 or SOCS3 (figure 5b). CCL26 (eotaxin-3) mRNA is upregulated in vitro by IL-13 stimulation and in bronchial biopsies of asthma patients with high levels of Th2 inflammation in their airways [25]. CCL26 mRNA upregulation was confirmed in BEAS2-B cultures stimulated with IL-13 (figure 5c). To investigate the role of SOCS1 in the regulation of 

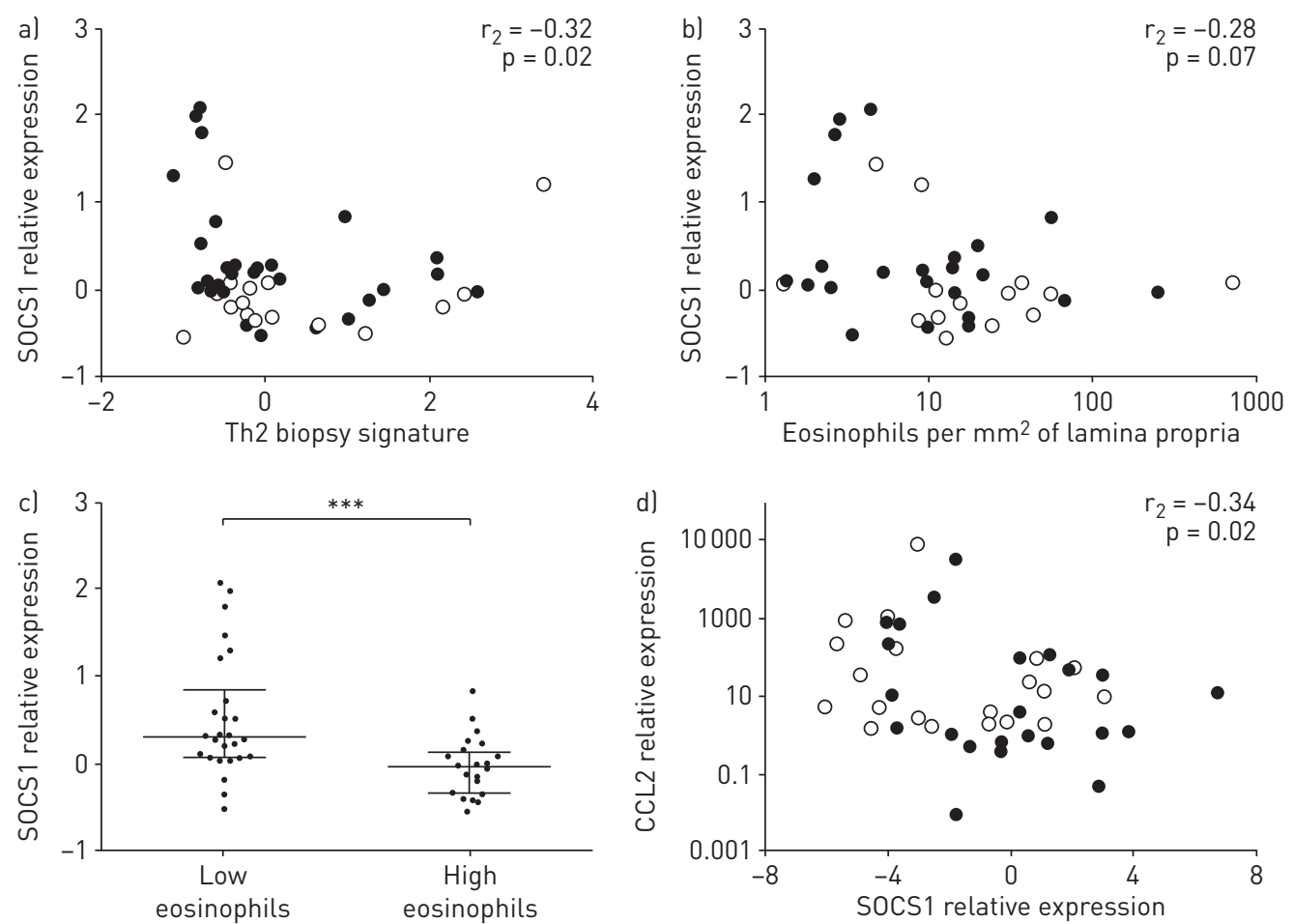

FIGURE 3 Suppressor of cytokine signalling (SOCS)1 gene expression is decreased in patients with persistent T-helper type 2 (Th2) inflammation and eosinophilia in the airways. a) SOCS1 and the Th2 biopsy signature $(n=47)$ and b) SOCS1 and eosinophil numbers in the lamina propria $(n=40)$ showed a negative correlation. c) SOCS1 expression was decreased in subjects with high airway eosinophilia $1 \geqslant 9.2$ eosinophils $/ \mathrm{mm}^{2}$ lamina propria, $\mathrm{n}=26$ ) compared to those with low airway eosinophilia $\left(<9.2\right.$ eosinophils per $\mathrm{mm}^{2}$ lamina propria; $\mathrm{n}=26)$. d) SOCS1 and CCL26 expression in the airway showed a significant negative correlation $(\mathrm{n}=44)$. Black circles represent mild/moderate asthma patients and white circles represent severe asthma patients. MannWhitney U-test and Spearman's Rank tests were employed for statistical analysis. Data are presented as median (interquartile range). ${ }^{* * *}: \mathrm{p}<0.001$.

IL-13-induced CCL26, SOCS1 was overexpressed in BEAS-2B cells using a Myc-tagged SOCS1 plasmid. Transfection with the SOCS1 plasmid lead to overexpression of SOCS1 in the cells compared with the empty vector control (figure 5d). Next, cells were transfected with either the empty vector or the Myc-tagged SOCS1 plasmid for $24 \mathrm{~h}$, then stimulated with IL-13 for a further $24 \mathrm{~h}$. A significant decrease in CCL26 mRNA was observed in the SOCS1 overexpressing cells (figure 5d).

We then wished to confirm these observations in primary bronchial epithelial cells (PBECs). PBECs were stimulated with IL-13 (50 ng.mL $\left.{ }^{-1}\right)$ over a 24-h time course. SOCS1 mRNA was again rapidly up-regulated $2 \mathrm{~h}$ after stimulation and remained elevated over the 24-h time course and SOCS2 and SOCS3 mRNA were not induced (figure 6a). As anticipated, CCL26 mRNA was also induced in IL-13-stimulated cells (figure 6b). PBECs were transfected using electroporation (transfection efficiencies were routinely greater than $55 \%$ as shown in figure E7) and again, overexpression of SOCS1 reduced IL-13 induction of CCL26 mRNA compared with empty vector control (figure 6c).

\section{Discussion}

Severe asthma affects up to $10 \%$ of all asthma patients, but the underlying mechanisms causing persistent eosinophilic inflammation, despite high-dose inhaled corticosteroid treatment, are yet to be fully elucidated $[10,27]$. SOCS molecules have been shown to regulate Th2 cytokine signalling both in vitro and in in vivo mouse models of allergic airway inflammation; however, it is unclear if similar regulatory mechanisms exist in human asthma.

In this study, we investigated, for the first time, the expression of SOCS1, SOCS2 and SOCS3 in the airways of healthy controls, mild/moderate asthmatics and severe asthmatics. We showed that patients with severe asthma and persistent eosinophilic airway inflammation have decreased epithelial SOCS1 gene expression compared with patients with milder more stable asthma. This reduced SOCS1 mRNA was inversely associated with an IL-13-dependent Th2 gene signature and tissue eosinophilia, which appears paradoxical, as one would anticipate IL-13 signalling to be associated with increased SOCS1 expression in an attempt to "switch off" IL-13 driven responses. It is worth commenting that baseline SOCS1 gene 
a)

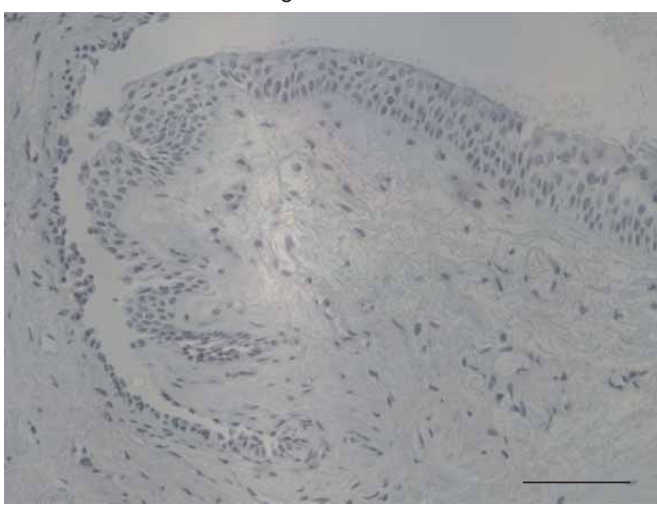

c)

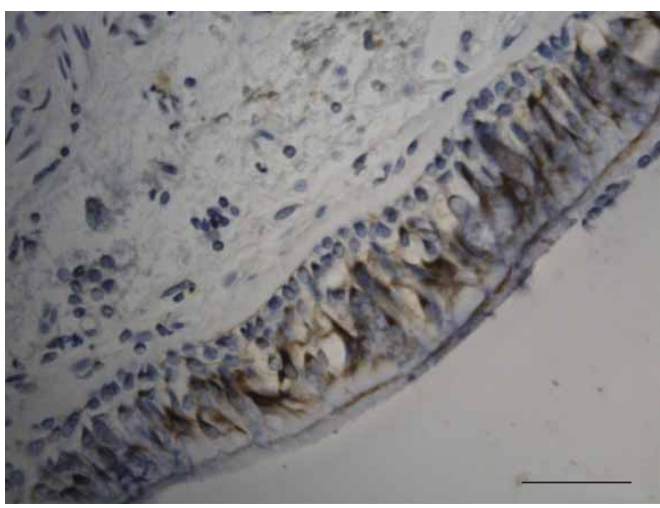

b)

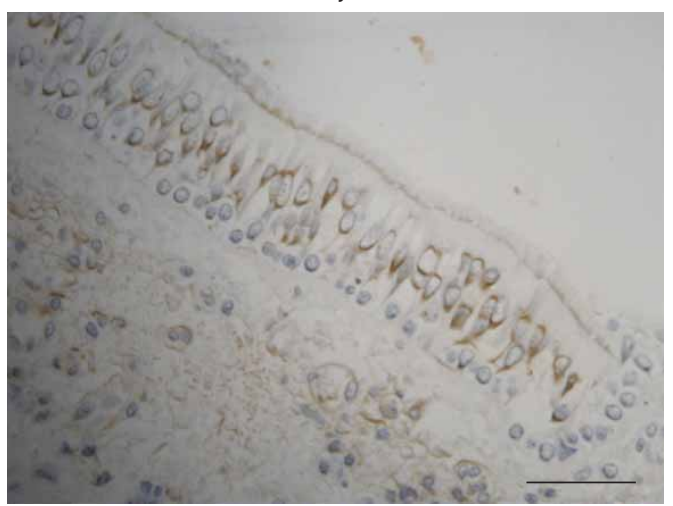

d)

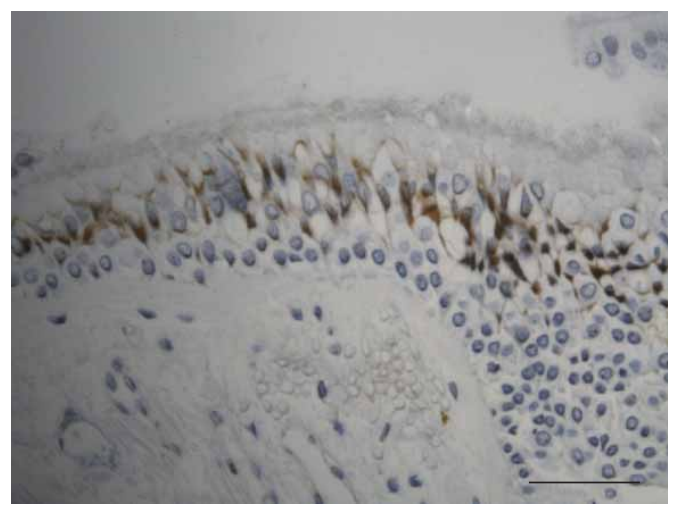

FIGURE 4 Localisation of suppressor of cytokine signalling (SOCS)1 protein predominantly to the bronchial epithelium. a) Negative control for SOCS1 staining. Glycol methacrylate-embedded bronchial biopsy sections from b) healthy controls, c) mild/moderate asthmatics and d) severe asthmatics were stained for SOCS1 using immunohistochemistry. Staining showed localisation of SOCS1 to the bronchial epithelium as depicted by the brown staining. Representative images show negative control staining and epithelial SOCS1 biopsy staining. Scale bars $=100 \mu \mathrm{m}$.

expression in healthy subjects is low (and not different from severe asthmatics), presumably reflecting the absence of pro-inflammatory cytokine stimuli and thus no requirement for the negative feedback regulator. The key difference is that in the severe asthma population with persistent Th2 signalling and eosinophilia, the failure of SOCS1 mRNA up-regulation is inappropriate.

This effect appears specific for SOCS1, as we identified no differences in SOCS2 or SOCS3 gene expression in relation to disease severity, Th2 signature, or eosinophilic inflammatory infiltrate. SOCS2 and SOCS3 have been implicated in animal models of asthma and SOCS molecules can interact with each other. For example, SOCS2 has been shown to regulate the expression of SOCS1 and SOCS3 via direct interactions [17] and SOCS2 knockout mice were shown to have elevated levels of SOCS1 and SOCS3 in $\mathrm{T}$ cells [18]. However, our data examining the gene expression of SOCS molecules in human asthma suggest a specific dysregulation of SOCS1 in severe asthma with persistent Th2 inflammation despite high-dose inhaled corticosteroids. One limiting factor of our study is the cross-sectional design; therefore, we cannot comment on the dynamic changes in SOCS expression. However, the pattern of specific SOCS1 down-regulation was consistent across severe asthma patients with persistent eosinophilia thus suggesting that it is unlikely to be a transient feature.

We utilised immunohistochemistry to examine localisation of SOCS proteins and better inform our subsequent in vitro mechanistic analysis. SOCS1 was found to be localised predominantly to the bronchial epithelium of the airway tissue. This is consistent with previous data, whereby mice challenged intra-tracheally with IL-13 displayed SOCS1 induction localised to the airway epithelium [15]. Future work to quantify SOCS1 protein in bronchial epithelial cells of healthy controls, mild/moderate and severe asthma patients would be valuable.

In vitro studies and in vivo animal models have shown SOCS1 attenuates Th2 inflammation. Local airway induction of SOCS1 in response to IL-13 administration in an in vivo mouse model was associated with 

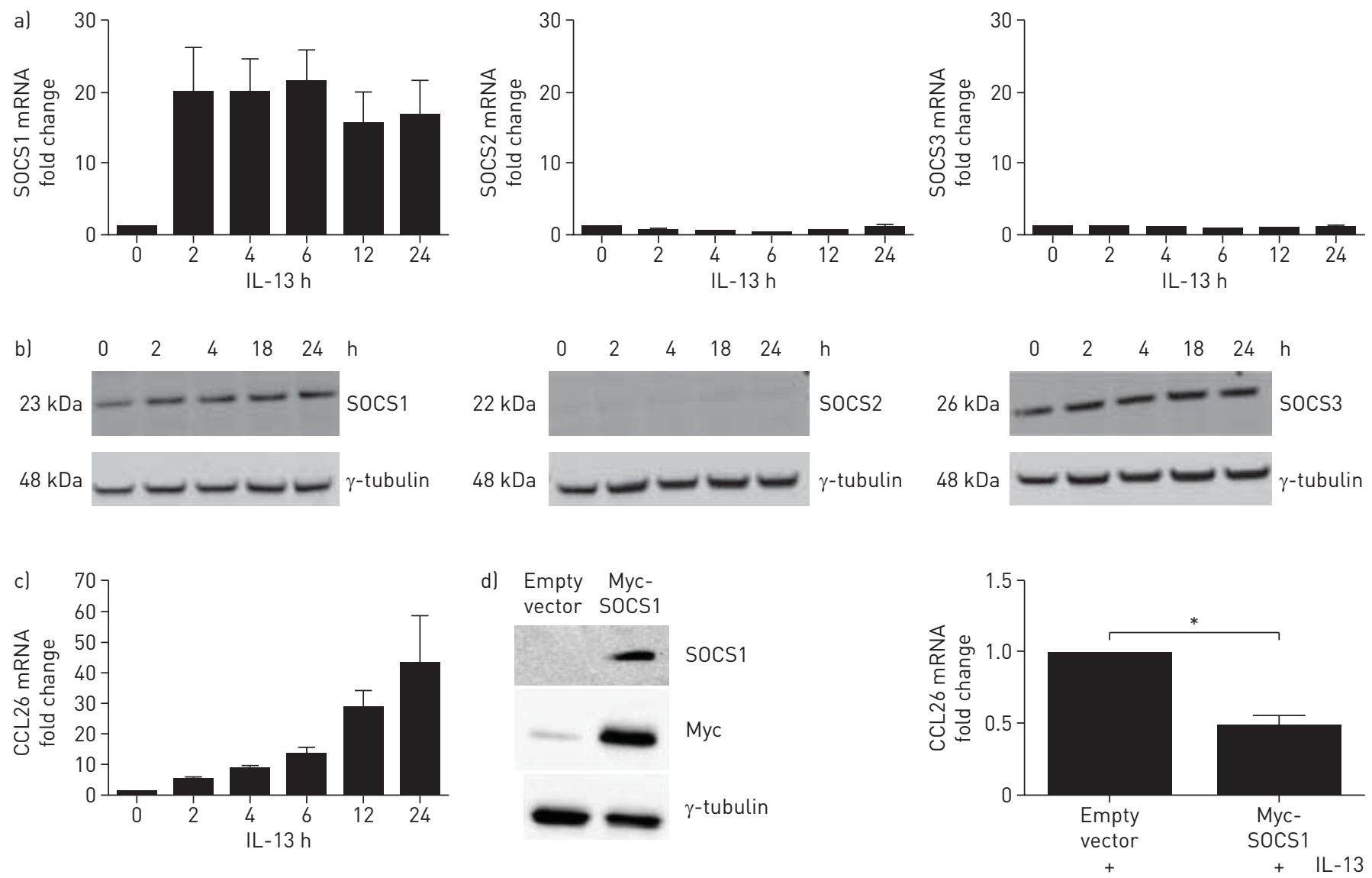

FIGURE 5 Suppressor of cytokine signalling (SOCS)1 overexpression in BEAS-2B cultures inhibited interleukin (IL)-13-induced CCL26 mRNA. a) Cells stimulated with IL-13 (50 ng. $\left.\mathrm{mL}^{-1}\right)$ over $24 \mathrm{~h}$ showed upregulation of SOCS1 mRNA but not SOCS2 or SOCS3 mRNA (n=3). b) Cells stimulated with IL-13 (50 ng. $\left.\mathrm{ml}^{-1}\right)$ over $24 \mathrm{~h}$ showed upregulation of SOCS1 protein but not SOCS2 or SOCS3 protein. c) Cells stimulated with IL-13 (50 ng. $\mathrm{mL}^{-1}$ ) over $24 \mathrm{~h}$ showed upregulation of CCL26 mRNA. d) Cells were transfected with an empty vector or S0CS1 overexpression plasmid then probed for SOCS1 and the plasmid Myc-tag to confirm SOCS1 overexpression. Cells were then treated with an empty vector or SOCS1 overexpression plasmid for $24 \mathrm{~h}$, stimulated with IL-13 for a further $24 \mathrm{~h}$ then CCL26 mRNA was measured using quantitative PCR. Data are presented as median (interquartile range). The Mann-Whitney U-test was employed for statistical analysis ( $n=4)$. *: $p<0.05$.

suppression of eotaxin levels [15]. In another study, an ovalbumin-induced airway inflammation model was utilised and $\mathrm{SOCS}^{-1-}$ IFN- $\gamma^{-/-}$mice had increased eosinophilic infiltration in the lungs and elevated Th2 cytokines compared to IFN- $\gamma^{-1-}$ control mice [14]. These animal studies support our data in human asthma whereby reduced SOCS1 expression in severe asthma is also associated with airway eosinophilia and an IL-13-dependent Th2 gene signature, suggesting epithelial SOCS1 has a central role in regulating IL-13 signalling in the airway and airway eosinophilia. Indeed high SOCS1 expression in airway eosinophils has been reported to inhibit their response to the IL-5 family cytokines [28]. Therefore SOCS1 deficiency in eosinophils in the airway of severe asthma patients may cause increased sensitivity to IL-5 cytokine signalling leading to enhanced eosinophil survival and pathologies in the airway.

IL-13 is a Th2 cytokine that has been implicated in human asthma and is elevated in severe asthma subjects with persistent eosinophilia [10, 29]. IL-13 signals via the STAT6 pathway and the absolute requirement of SOCS1 in attenuating Th2 inflammation via inhibition of STAT6 phosphorylation has been observed in SOCS1 deficient mouse embryonic fibroblasts $[15,30]$. As SOCS1 was localised to the bronchial epithelium of bronchial biopsies, we wished to examine the role of SOCS1 in regulating IL-13 epithelial signalling in vitro using the bronchial epithelial BEAS-2B cell line and primary bronchial epithelial cells (PBECs). CCL26 (eotaxin-3) expression was examined as a downstream readout of IL-13 signalling as previous studies have found it to be one of the most highly upregulated genes in the airways of asthma patients with Th2 inflammation, it is IL-13 dependent and a potent eosinophil chemoattractant [25, 31, 32]. CCL26 is most highly induced in response to IL-13 in bronchial epithelial cells from severe asthma patients compared to mild patients and this correlates with increased airway eosinophils [33]. Cells stimulated with IL-13 showed SOCS1 mRNA was rapidly induced but this was not the case for SOCS2 or SOCS3. This selective induction of SOCS1 further supports its role in inhibiting IL-13 driven Th2 inflammation and is consistent with our 

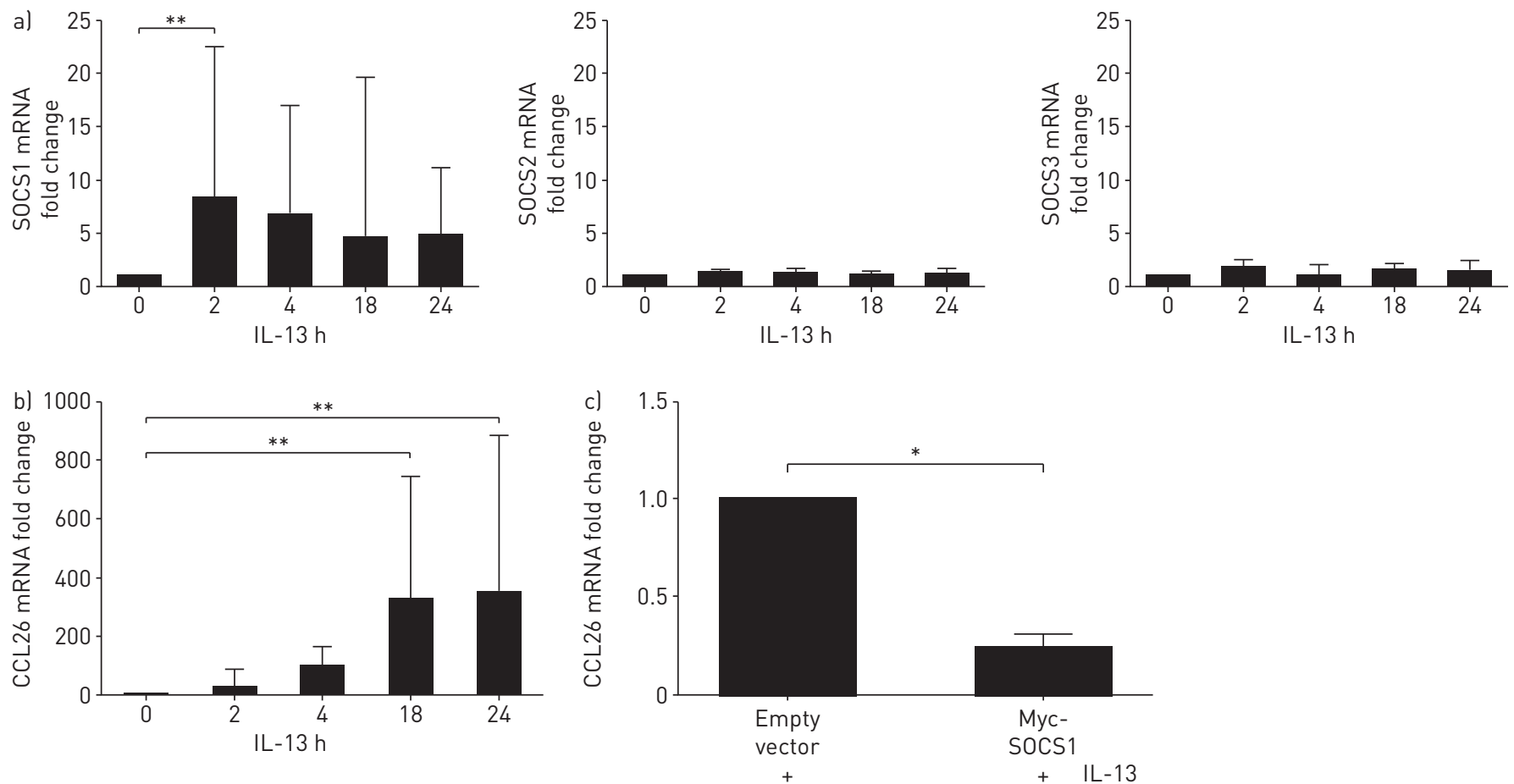

FIGURE 6 Suppressor of cytokine signalling (SOCS)1 overexpression in primary bronchial epithelial cells (PBEC) cultures inhibited interleukin (IL)-13-induced CCL26 mRNA. a) Cells stimulated with IL-13 (50 ng. $\left.\mathrm{mL}^{-1}\right)$ over $24 \mathrm{~h}$ showed upregulation of SOCS1 mRNA but not SOCS2 or SOCS3 mRNA (n=5). b) Cells stimulated with IL-13 (50 $\left.\mathrm{ng} \cdot \mathrm{mL}^{-1}\right)$ over $24 \mathrm{~h}$ showed upregulation of CCL26 mRNA. c) Cells were transfected with an empty vector or SOCS1 overexpression plasmid for $24 \mathrm{~h}$ and stimulated with IL-13 for a further $24 \mathrm{~h}$ then CCL26 mRNA was measured using quantitative PCR. Data are presented as median (interquartile range). Kruskal-Wallis and t-tests were employed for statistical analysis ( $\mathrm{n}=3$ ). $*: p<0.05 ; * *: p<0.01$.

patient data showing no relationship between SOCS2 or SOCS3 expression and the IL-13 dependent gene signature or airway eosinophilia. As anticipated, and consistent with other systems, CCL26 mRNA was induced in BEAS-2B cells and PBECs in response to IL-13. Consistent with the role of SOCS1 regulating IL-13 signalling, when SOCS1 was over-expressed in BEAS-2B and PBEC cultures, IL-13 induction of CCL26 was inhibited. We have previously reported in this same cohort of severe asthmatics, that patients with an elevated IL-13 dependent-Th2 gene signature, have increased numbers of IL- $13^{+}$cells infiltrating the epithelium [10]. Taken together, these patients with severe asthma, despite being on high dose inhaled corticosteroid treatment, have persistent airway eosinophilia, increased intra-epithelial IL- $13^{+}$cells, persistent IL-13 signalling in the airway (Th2 gene signature) and decreased SOCS1 expression. This suggests a breakdown in regulation of IL-13 by SOCS1 in these patients and it will be of interest to assess whether SOCS1 expression is modified upon IL-13 inhibition, for example, after treatment with IL-13 antagonists such as those currently in phase $2 / 3$ clinical trials $[28,34,35]$.

A study assessing functional variants of SOCS1 within a population of adult Japanese asthma patients found a significant association between the SOCS1 promoter polymorphism, $-1478 \mathrm{CA}<\mathrm{del}$, and adult asthma. It is suggested this promoter polymorphism leads to increased SOCS1 and inhibition of interferons (IFNs) leading to higher susceptibility to virus-induced asthma exacerbations [36]. Another study has recently shown that increased nuclear SOCS1 in asthmatic bronchial epithelium suppresses rhinovirus induction of innate IFNs [37]. However, our data in severe asthmatics would suggest that reduced SOCS1 in the epithelium is associated with persistent eosinophilia, which is another major risk factor for exacerbations in this population [38].

In summary, we have investigated the expression of SOCS1, SOCS2 and SOCS3 within the airways of healthy controls, mild/moderate asthmatics, and severe asthmatics. We found that SOCS1 gene expression was significantly decreased in the airways of severe asthma patients when compared to mild/moderate asthma patients. Subjects with persistent eosinophilia and increased Th2 inflammation within the airway have decreased epithelial SOCS1 mRNA expression. In vitro investigation of BEAS-2B and primary bronchial epithelial cell cultures showed that SOCS1 was induced in response to IL-13 stimulation and SOCS1 overexpression led to reduced CCL26 mRNA induction in response to IL-13 stimulation. Further work will focus on why there is an inappropriate failure to upregulate SOCS1 in the epithelium of patients with severe asthma despite persistent Th2 signalling in the airway. 


\section{Acknowledgements}

We would like to thank Lorcan McGarvey, Queens University Belfast (Belfast, UK), for providing us with primary bronchial epithelial cells.

\section{References}

1 Masoli M, Fabian D, Holt S, et al. The global burden of asthma: executive summary of the GINA Dissemination Committee report. Allergy 2004; 59: 469-478.

2 British Thoracic Society Scottish Intercollegiate Guidelines Network. British Guideline on the Management of Asthma. Thorax 2008; 63: Suppl 4, iv1-iv121.

Wenzel S. Severe asthma in adults. Am J Respir Crit Care Med 2005; 172: 149-160.

Holgate ST. Innate and adaptive immune responses in asthma. Nat Med 2012; 18: 673-683.

Szefler SJ, Martin RJ, King TS, et al. Significant variability in response to inhaled corticosteroids for persistent asthma. J Allergy Clin Immunol 2002; 109: 410-418.

6 Haldar P, Pavord ID, Shaw DE, et al. Cluster analysis and clinical asthma phenotypes. Am J Respir Crit Care Med 2008; 178: 218-224.

7 Moore WC, Meyers DA, Wenzel SE, et al. Identification of asthma phenotypes using cluster analysis in the Severe Asthma Research Program. Am J Respir Crit Care Med 2010; 181: 315-323.

8 Woodruff PG, Modrek B, Choy DF, et al. T-helper type 2-driven inflammation defines major subphenotypes of asthma. Am J Respir Crit Care Med 2009; 180: 388-395.

9 Jia G, Erickson RW, Choy DF, et al. Periostin is a systemic biomarker of eosinophilic airway inflammation in asthmatic patients. J Allergy Clin Immunol 2012; 130: 647-654.

10 Shikotra A, Choy DF, Ohri CM, et al. Increased expression of immunoreactive thymic stromal lymphopoietin in patients with severe asthma. J Allergy Clin Immunol 2012; 129: 104-111.

11 Wills-Karp M, Luyimbazi J, Xu X, et al. Interleukin-13: central mediator of allergic asthma. Science 1998; 282 : 2258-2261.

12 Elliott J, Johnston JA. SOCS: role in inflammation, allergy and homeostasis. Trends Immunol 2004; 25: 434-440.

13 Starr R, Willson TA, Viney EM, et al. A family of cytokine-inducible inhibitors of signalling. Nature 1997; 387: 917-921.

14 Lee C, Kolesnik TB, Caminschi I, et al. Suppressor of cytokine signalling 1 (SOCS1) is a physiological regulator of the asthma response. Clin Exp Allergy 2009; 39: 897-907.

15 Fukuyama S, Nakano T, Matsumoto T, et al. Pulmonary suppressor of cytokine signaling-1 induced by IL-13 regulates allergic asthma phenotype. Am J Respir Crit Care Med 2009; 179: 992-998.

16 Metcalf D, Greenhalgh CJ, Viney E, et al. Gigantism in mice lacking suppressor of cytokine signalling-2. Nature 2000; 405: 1069-1073.

17 Piessevaux J, Lavens D, Montoye T, et al. Functional cross-modulation between SOCS proteins can stimulate cytokine signaling. J Biol Chem 2006; 281: 32953-32966.

18 Knosp CA, Carroll HP, Elliott J, et al. SOCS2 regulates T helper type 2 differentiation and the generation of type 2 allergic responses. J Exp Med 2011; 208: 1523-1531.

19 Lopez E, Zafra MP, Sastre B, et al. Suppressors of cytokine signaling 3 expression in eosinophils: regulation by PGE(2) and Th2 cy5okines. Clin Dev Immunol 2011; 2011: 917015.

20 Seki $\mathrm{Y}$, Inoue $\mathrm{H}$, Nagata $\mathrm{N}$, et al. SOCS-3 regulates onset and maintenance of $\mathrm{T}(\mathrm{H}) 2$-mediated allergic responses. Nat Med 2003; 9: 1047-1054.

21 Butler CA, McQuaid S, Taggart CC, et al. Glucocorticoid receptor beta and histone deacetylase 1 and 2 expression in the airways of severe asthma. Thorax 2012; 67: 392-398.

22 McGarvey LP, Butler CA, Stokesberry S, et al. Increased expression of bronchial epithelial transient receptor potential vanilloid 1 channels in patients with severe asthma. J Allergy Clin Immunol 2014; 133: 704-712.

23 Chung KF, Wenzel SE, Brozek JL, et al. International ERS/ATS guidelines on definition, evaluation and treatment of severe asthma. Eur Respir J 2014; 43: 343-373.

24 Bradding P, Feather $\mathrm{IH}$, Howarth PH, et al. Interleukin 4 is localized to and released by human mast cells. J Exp Med 1992; 176: 1381-1386.

25 Choy DF, Modrek B, Abbas AR, et al. Gene expression patterns of Th2 inflammation and intercellular communication in asthmatic airways. J Immunol 2011; 186: 1861-1869.

26 Schmittgen TD, Livak KJ. Analyzing real-time PCR data by the comparative C(T) method. Nat Protoc 2008; 3 : 1101-1108.

27 Green RH, Brightling CE, McKenna S, et al. Asthma exacerbations and sputum eosinophil counts: a randomised controlled trial. Lancet 2002; 360: 1715-1721.

28 Burnham ME, Koziol-White CJ, Esnault S, et al. Human airway eosinophils exhibit preferential reduction in STAT signaling capacity and increased CISH expression. J Immunol 2013; 191: 2900-2906.

29 Corren J, Lemanske RF, Hanania NA, et al. Lebrikizumab treatment in adults with asthma. N Engl J Med 2011; 365: 1088-1098.

30 Sato T, Saito R, Jinushi T, et al. IFN- $\gamma$-induced SOCS-1 regulates STAT6-dependent eotaxin production triggered by IL-4 and TNF- $\alpha$. Biochem Biophys Res Commun 2004; 314: 468-475.

31 Kitaura M, Suzuki N, Imai T, et al. Molecular cloning of a novel human CC chemokine (Eotaxin-3) that is a functional ligand of CC chemokine receptor 3. J Biol Chem 1999; 274: 27975-27980.

32 Provost V, Larose MC, Langlois A, et al. CCL26/eotaxin-3 is more effective to induce the migration of eosinophils of asthmatics than CCL11/eotaxin-1 and CCL24/eotaxin-2. J Leukoc Biol 2013; 94: 213-222.

33 Larose MC, Chakir J, Archambault AS, et al. Correlation between CCL26 production by human bronchial epithelial cells and airway eosinophils: Involvement in patients with severe eosinophilic asthma. J Allergy Clin Immunol 2015; 136: 904-913.

34 Piper E, Brightling C, Niven R, et al. A phase II placebo-controlled study of tralokinumab in moderate-to-severe asthma. Eur Respir J 2013; 41: 330-338.

35 Wenzel S, Ford L, Pearlman D, et al. Dupilumab in persistent asthma with elevated eosinophil levels. $N$ Engl J Med 2013; 368: 2455-2466. 
36 Harada M, Nakashima K, Hirota T, et al. Functional polymorphism in the suppressor of cytokine signaling 1 gene associated with adult asthma. Am J Respir Cell Mol Biol 2007; 36: 491-496.

37 Gielen V, Sykes A, Zhu J, et al. Increased nuclear suppressor of cytokine signaling 1 in asthmatic bronchial epithelium suppresses rhinovirus induction of innate interferons. J Allergy Clin Immunol 2015; 136: 177-188.

38 Haldar P, Brightling CE, Hargadon B, et al. Mepolizumab and exacerbations of refractory eosinophilic asthma. N Engl J Med 2009; 360: 973-984. 